\title{
Cytotoxic necrotizing factor 1 promotes bladder cancer angiogenesis through activating $\mathrm{RhoC}$
}

\author{
Yaxiu Guo $^{1}$ | Jingyu Wang ${ }^{1}$ | Kaichen Zhou ${ }^{1}$ | Junqiang Lv ${ }^{1}$ ～Lei Wang ${ }^{2}$ | \\ Shan Gao $^{2}$ | Evan T. Keller ${ }^{3}$ | Zhi-Song Zhang ${ }^{2}$ | Quan Wang ${ }^{1}$ | Zhi Yao ${ }^{1,4}$
}

\author{
${ }^{1}$ Department of Immunology, Key Laboratory of Immune Microenvironment and Disease of the Educational Ministry of China, Tianjin Key Laboratory of \\ Cellular and Molecular Immunology, School of Basic Medical Sciences, Tianjin Medical University, Tianjin, China \\ ${ }^{2}$ State Key Laboratory of Medicinal Chemical Biology and College of Pharmacy, Collaborative Innovation Center for Biotherapy, Tianjin Key Laboratory of \\ Molecular Drug Research, Nankai University, Tianjin, China \\ ${ }^{3}$ Department of Urology, University of Michigan, Ann Arbor, MI, USA \\ ${ }^{4} 2011$ Collaborative Innovation Center of Tianjin for Medical Epigenetics, Tianjin Medical University, Tianjin, China
}

\begin{abstract}
Correspondence
Zhi-Song Zhang, State Key Laboratory of Medicinal Chemical Biology and College of Pharmacy, Collaborative Innovation Center for Biotherapy, and Tianjin Key Laboratory of Molecular Drug Research, Nankai University, Tianjin 300350, China.

Email: zzs@nankai.edu.cn

Quan Wang and Zhi Yao, Department of Immunology, Key Laboratory of Immune Microenvironment and Disease of the Educational Ministry of China, Tianjin Key Laboratory of Cellular and Molecular Immunology, School of Basic Medical Sciences, Tianjin Medical University, Tianjin 300070, China.

Email: wangquan@tmu.edu.cn (Q.W.) and yaozhi@tmu.edu.cn (Z. Y.)
\end{abstract}

\section{Funding information}

National Natural Science Foundation of China, Grant/Award Number: 31970133 , 81672740 and 81572882

\begin{abstract}
Uropathogenic Escherichia coli (UPEC), a leading cause of urinary tract infections, is associated with prostate and bladder cancers. Cytotoxic necrotizing factor 1 (CNF1) is a key UPEC toxin; however, its role in bladder cancer is unknown. In the present study, we found CNF1 induced bladder cancer cells to secrete vascular endothelial growth factor (VEGF) through activating Ras homolog family member C (RhoC), leading to subsequent angiogenesis in the bladder cancer microenvironment. We then investigated that CNF1-mediated RhoC activation modulated the stabilization of hypoxia-inducible factor $1 \alpha(\mathrm{HIF} 1 \alpha)$ to upregulate the VEGF. We demonstrated in vitro that active RhoC increased heat shock factor 1 (HSF1) phosphorylation, which induced the heat shock protein $90 \alpha$ (HSP90 $\alpha$ ) expression, leading to stabilization of HIF $1 \alpha$. Active RhoC elevated HSP90 $\alpha$, HIF1 $\alpha$, VEGF expression, and angiogenesis in the human bladder cancer xenografts. In addition, HSP90 $\alpha$, HIF1 $\alpha$, and VEGF expression were also found positively correlated with the human bladder cancer development. These results provide a potential mechanism through which UPEC contributes to bladder cancer progression, and may provide potential therapeutic targets for bladder cancer.
\end{abstract}

\section{K E Y W O R D S}

angiogenesis, bladder cancer, cytotoxic necrotizing factor 1, RhoC, uropathogenic Escherichia coli

\footnotetext{
Abbreviations: ANOVA, analysis of variance; Co-IP, co-immunoprecipitation; DAPI, 2-(4-amidinophenyl)-1H-indole-6-carboxamidine; ELISA, enzyme-linked immunosorbent assay; FITC, fluorescein isothiocyanate; H\&E, hematoxylin and eosin; HRP, horseradish peroxidase; IHC, immunohistochemistry; OCT, optimum cutting temperature; PBS, phosphate-buffered saline; PEI, polyethylenimine; qRT-PCR, quantitative real-time reverse transcription polymerase chain reaction; RPMI, Roswell Park Memorial Institute.
}

Yaxiu Guo and Jingyu Wang contributed equally to this study. 


\section{1 | INTRODUCTION}

Uropathogenic Escherichia coli (UPEC)-induced urinary tract infections (UTIs) usually evoke cystitis, pyelonephritis, and prostatitis. ${ }^{1-3}$ In addition, UPEC was also reported to accelerate the prostate cancer progression in the genetically engineered Hi-Myc mouse prostate cancer model, and increase the risk of bladder cancer through promoting CDKN2A methylation. ${ }^{4,5}$ Previously, we reported that cytotoxic necrotizing factor 1 (CNF1), a key UPEC toxin, promoted migration and invasion of prostate cancer cells and prostate cancer metastasis by activating the Cdc42-PAK1 Axis. ${ }^{6}$ Although UPEC frequently infects bladder, the role of CNF1 in bladder cancer has not been reported.

CNF1 binds to cells through a specific receptor, enters the cytosol, and then activates the Rho GTPases (including RhoA, Cdc42, and Rac1) by deamidation of specific glutamine residues. ${ }^{7}$ RhoA, RhoB, and RhoC belong to the same subfamily of Rho GTPases based on phylogenetic analysis, ${ }^{8}$ and the expression or activity of RhoA and RhoC are increased in many types of human tumors. ${ }^{9,10} \mathrm{RhoC}$ has been reported to facilitate the invasion and metastasis of tumor cells ${ }^{11}$ and promote the tumor angiogenesis by inducing the release of vascular endothelial growth factor (VEGF). ${ }^{12}$ However, the mechanism by which RhoC induces VEGF is unclear.

Angiogenesis is essential for tumor progression and is highly dependent on VEGF, ${ }^{13}$ which is expressed by most of the malignant tumors. ${ }^{14,15}$ Under hypoxic conditions, hypoxia-inducible factor 1 (HIF1) induces the transcription of VEGF. ${ }^{16}$ HIF1 is a heterodimeric transcription factor composed of a constitutively expressed HIF $1 \beta$-subunit and an $\mathrm{O}_{2}$-regulated HIF1 $\alpha$ subunit, and is a major regulator of angiogenesis in the tumor microenvironment. ${ }^{17,18}$

Under normoxic conditions, HIF1 $\alpha$ can be hydroxylated at critical proline residues by dioxygenase prolyl hydroxylases, and degraded through pVHL-mediated ubiquitin-proteasome pathway. ${ }^{19,20}$ However, under hypoxic conditions, the activity of dioxygenase prolyl hydroxylases is suppressed, and HIF $1 \alpha$ is stable. ${ }^{21}$ In addition, HIF1 $\alpha$ degradation can be decreased by several pVHL-independent mechanisms. ${ }^{22,23}$ For example, the heat shock protein 90 (HSP90), a molecular chaperone, has been reported to stabilize HIF1 $\alpha$ by binding it directly. ${ }^{22}$ The role and mechanism of RhoC in regulating HIF1 $\alpha$ has not been reported.

In this study, we examined if and how CNF1 facilitated VEGF secretion by tumor cells and the subsequent angiogenesis in the bladder cancer microenvironment. We found that CNF1 induced VEGF and angiogenesis through RhoCdependent activation of the HSF1-HSP90 $\alpha$-HIF1 $\alpha$ axis. These results may provide potential bladder cancer therapeutic targets.

\section{2 | MATERIALS AND METHODS}

\section{1 | Cell lines}

The T24, 5637, UMUC3, and 293T cells were obtained from the ATCC (Manassas, VA, USA). The HUVECs were a generous gift from Dr Zhi-Song Zhang from the College of Pharmacy, Nankai University, Tianjin, China. For hypoxic conditions, the cells were cultured in a modular incubator chamber flushed with mixed gas consisting of $1 \% \mathrm{O}_{2}, 5 \%$ $\mathrm{CO}_{2}$, and $94 \% \mathrm{~N}_{2}$ at $37^{\circ} \mathrm{C}$ for normoxic conditions, mixed gas consisting of $20 \% \mathrm{O}_{2}, 5 \% \mathrm{CO}_{2}$, and $75 \% \mathrm{~N}_{2}$ at $37^{\circ} \mathrm{C}$ was used. Other reagents used included the HSF1 inhibitor KRIBB11 (S8402; Selleck Chemicals, Houston, TX, USA); the NF- $\mathrm{kB}$ inhibitor BAY 11-7085 (HY-10257; MedChem Express, Monmouth Junction, NJ, USA); and the proteasome inhibitor MG132 (M7449; Sigma-Aldrich, St. Louis, MO, USA).

\section{2 | Plasmids}

The cnfl gene from UPEC strains 11 was amplified by PCR and cloned into pET-28a(+) (Novagen, Madison, WI, USA). The coding sequences for RhoC, HIF1 $\alpha$, and HSP90 $\alpha$ were subcloned into pCMV-Tag2B (Stratagene, La Jolla, CA, USA), pLVX-EF1 $\alpha$-IRES-Puro, pLVXIRES-Hyg (Clontech, Mountain View, CA, USA) or pCDNA3.1-HA-Hygro (Invitrogen, Carlsbad, CA, USA). The constructed plasmids and primer sequences are listed in Tables $\mathrm{S} 1$ and $\mathrm{S} 2$.

\section{3 | CNF1 recombinant protein expression and purification}

Recombinant proteins of CNF1 and its mutant C866S were expressed, purified, and identified as described previously. ${ }^{6}$

\section{4 | Mutagenesis}

Mutagenesis of cnfl and RhoC was performed using a Fast Mutagenesis System kit (TransGen Biotech, Beijing, China) according to the manufacturer's protocol. The primers used for mutation are listed in Table S2.

\section{5 | Transwell migration and invasion assays}

Cell migration and invasion assays were performed using Transwell chambers (pore size $8 \mu \mathrm{m}$, Costar, Corning 3422), 
with Corning Matrigel Matrix (Corning Incorporated, Corning, NY, USA) for invasion assays. Cells $\left(1 \times 10^{5}\right.$ in $200 \mu \mathrm{L})$ resuspended in serum-free RPMI 1640 medium were added to the upper chamber of uncoated (for migration assays) or Matrigel-coated (for invasion assays) membranes with PBS, CNF1 (1 nmol/L), or C866S (1 nmol/L). Then, $600 \mu \mathrm{L}$ of medium containing $20 \%$ fetal bovine serum with according PBS, CNF1 ( $1 \mathrm{nmol} / \mathrm{L})$, or C866S $(1 \mathrm{nmol} / \mathrm{L})$ was added to the lower chamber. After 12 hours (migration assays) or 24 hours (invasion assays) incubation at $37{ }^{\circ} \mathrm{C}$ in a $5 \% \mathrm{CO}_{2}$ humidified atmosphere, the cells that adhered to the bottom surface of the inserts were fixed in $4 \%$ paraformaldehyde for 1 hour, and stained with $0.1 \%$ crystal violet (Beijing Solarbio Science \& Technology Co. Ltd., Beijing, China) for 15 minutes. Finally, the filters were washed three times in PBS and images were captured under a microscope (Leica Microsystems, Wetzlar, Germany) at 200x magnification.

\subsection{Gelatin zymography assay}

The activities of MMP2 and MMP9 were analyzed by gelatin zymography as described previously. ${ }^{24}$ In brief, cells were incubated with serum-free medium supplemented with PBS or CNF1 $(1 \mathrm{nmol} / \mathrm{L})$ for 24 hours at $37^{\circ} \mathrm{C}$. The conditioned medium was collected and mixed with $\beta$-mercaptoethanolfree sample loading buffer without boiling, and then electrophoresed on 10\% (w/v) SDS-PAGE containing 0.1\% (w/v) gelatin (Sigma- Aldrich). After electrophoresis, the gels were washed for 30 minutes with $2.5 \%(\mathrm{v} / \mathrm{v})$ Triton X-100 (Merck Millipore, Billerica, MA, USA) to remove SDS and then incubated overnight at $37^{\circ} \mathrm{C}$ in developing buffer $\left(50 \mathrm{mmol} / \mathrm{L}\right.$ Tris, pH 7.8, $\left.5 \mathrm{mmol} / \mathrm{L} \mathrm{CaCl}_{2}\right)$. Bands corresponding to the enzyme were visualized by staining with $0.2 \%(\mathrm{w} / \mathrm{v})$ Coomassie brilliant blue R-250 (Merck Millipore), 50\% (v/v) methanol, and 10\% (v/v) acetic acid. The activities of MMP2 and MMP9 were quantified using ImageJ and $\beta$-Actin was detected using western immunoblotting as an internal control.

\section{$2.7 \quad$ ELISA assay}

Human bladder cancer cells were seeded in 12-well culture plates and incubated with serum-free medium supplemented with PBS, CNF1 ( $3 \mathrm{nmol} / \mathrm{L})$, or CNF1 mutant C866S $\left(3 \mathrm{nmol} / \mathrm{L}\right.$ ) for the indicated times at $37^{\circ} \mathrm{C}$ under normoxic or hypoxic conditions. The medium was removed and stored at $-80^{\circ} \mathrm{C}$ until the assay was performed. VEGF in the medium was assayed using the Human VEGF Mini ABTS ELISA Development Kit (PeproTech, Rocky Hill, NJ, USA), according to the manufacturer's instructions.

\section{8 | Tube formation assay}

T24 and 5637 cells were cultured in serum-free RPMI 1640 supplemented with PBS or CNF1 ( $3 \mathrm{nmol} / \mathrm{L}$ ) for 36 hours. The conditioned media were collected separately, centrifuged, and stored at $-80^{\circ} \mathrm{C}$ Growth factor-reduced Matrigel (BD Biosciences, Bedford, MA, USA) was dissolved at $4^{\circ} \mathrm{C}$ and 96-well plates were prepared with $50 \mu \mathrm{L}$ of matrigel in each well after coating and incubating at $37^{\circ} \mathrm{C}$ for 1 hour. HUVECs $\left(1 \times 10^{4}\right)$ were suspended in $100 \mu \mathrm{L}$ of conditioned medium. After 6 hours of incubation at $37^{\circ} \mathrm{C}$, HUVECs tube formation was assessed using a photomicroscope, and each well was photographed at $100 \times$ magnification under a light microscope. The total length of the tubes was analyzed using the ImageJ software.

\section{9 | RNA isolation and qRT-PCR}

Total RNA was isolated using the TRIzol reagent (Beijing Solarbio Science \& Technology Co. Ltd.). Total RNA ( $2 \mu \mathrm{g})$ was used to synthesize first-strand cDNA using M-MuLV reverse transcriptase (Thermo Fisher Scientific, Waltham, MA, USA). qRT-PCR was then performed using the SYBR green mix (Thermo Fisher Scientific). The reactions were performed with a LightCycler 96 Real-Time PCR System (Roche, Basel, Switzerland). $\beta$-Actin was used as the endogenous control gene and the data were normalized based on the transcription level of $\beta$-Actin in the wild type and quantified using the comparative critical threshold cycle $2^{-\Delta \Delta C t}$ method. The primers used are listed in Table S2.

\subsection{0 | RNA sequencing}

After transfection with the vector or RhoC-Q63E, total RNA of T24 cells was extracted using the TRIzol reagent. The RNA sequencing (RNA-Seq) was performed by Majorbio (Shanghai, China). The data were analyzed on the free online platform of Majorbio I-Sanger Cloud Platform (www.isanger.com). The RNA-seq data are available under GEO accession GSE129295.

\subsection{Antibodies and western blotting}

Antibodies were ordered from the following companies: anti-HIF1 $\alpha$ (20960-1-AP, 1:1000), anti-HSF1 (51034-1AP, 1:1000), anti-RhoC (10632-1-AP, 1:1000), and antiMyc (60003-2-Ig, 1:2000) were from Proteintech (Chicago, IL,USA); anti-HSF1 phosphorylated at Ser326 antibody (ab76076, 1:2000) was from Abcam (Cambridge, MA, USA); anti-HA (\#3724, 1:1000), and anti-HSP90 $\alpha$ (\#8165, 1:1000) 
were from Cell Signaling Technology (Danvers, MA, USA); anti-RhoA (ARH04, 1:500) and anti-Rac1 (ARC03, 1:500) were from Cytoskeleton (Denver, CO, USA); anti-RhoB (sc8048, 1:500) was from Santa Cruz Biotechnology (Santa Cruz, CA, USA); anti-Cdc42 (BA2442, 1:500) was from Wuhan Boster Biological Technology Co. Ltd. (Wuhan, China); and anti-Flag (F1804, 1:1000) was from Sigma-Aldrich. Cells were washed with PBS three times after treatment. Whole cell lysates were prepared using RIPA lysis buffer (Merck Millipore), with the addition of complete protease inhibitors (Roche). The protein concentration was determined using the BCA Protein Assay Kit (Thermo Fisher Scientific) and approximately $20 \mu \mathrm{g}$ of cell lysates were used. Antibody binding was revealed using an HRP-conjugated anti-rabbit IgG or anti-mouse IgG (SigmaAldrich). Antibody complexes were detected using Immobilon Western Chemiluminescent HRP Substrate (Merck Millipore) and exposed in a Tanon-5200 machine.

\subsection{2 | Immunoprecipitation}

Cells were lysed using lysis buffer containing $50 \mathrm{mmol} / \mathrm{L}$ Tris, pH 7.4, $150 \mathrm{mmol} / \mathrm{L} \mathrm{NaCl}, 0.2 \mathrm{mmol} / \mathrm{L}$ EDTA, $1 \%$ NP-40, and protease inhibitors (Roche). The lysates were centrifuged at $14000 \mathrm{rpm}$ for $15 \mathrm{~min}$ at $4^{\circ} \mathrm{C}$. The extracts were incubated with Anti-c-Myc Agarose (Sigma-Aldrich) overnight at $4{ }^{\circ} \mathrm{C}$. The beads were then washed exhaustively with the lysis buffer. Immobilized proteins were eluted with $2 \times$ Laemmli sample buffer and subjected to SDS-PAGE.

\subsection{3 | RNA interference}

Double-stranded RNAs as siRNAs for the targeted genes and scrambled siRNA (siScr) were synthesized by GenePharma (Shanghai, China). The sequences (sense strand) of the siRNAs are listed in Table S2. Specific gene knockdowns were assessed by western blotting. Transfection of siRNAs was carried out using Lipofectamine 3000 (Invitrogen).

\subsection{4 | Rho GTPase activation assays}

T24 cells were seeded in $10-\mathrm{cm}$ dishes. After treatment, RhoC activation was measured using a Rho Activation Assay Biochem Kit (BK036, Cytoskeleton) according to the manufacturer's protocol.

\subsection{5 | Transduction and transfection}

T24 cells stably expressing RhoC constitutively active mutant Q63E were constructed using lentiviral supernatant.
T24 cells were transiently transfected with the RhoCrelated plasmids using Lipofectamine 3000 (Invitrogen). 293T cells were transiently transfected with the RhoC, HIF $1 \alpha$, and HSP90 $\alpha$-related plasmids using PEI (SigmaAldrich).

\subsection{6 | Xenograft model}

All animal studies were reviewed and approved by the Animal Care and Use Committee at Tianjin Medical University, Tianjin, China. We made every effort to minimize animal suffering and to reduce the number of animals used. Six- to eight-week-old female athymic BALB/c nude mice were purchased from the Academy of Military Medical Science (Beijing, China). T24 cells expressing RhoC mutant Q63E or the control were resuspended in $100 \mu \mathrm{L}$ of PBS and injected into the flank of the nude mice $\left(1 \times 10^{6}\right.$ cells per animal). The xenografts were measured as described in figure legends. Then the tumor was collected and the volume and weight were measured. The tumors were fixed in $10 \%$ formalin or OCT compound for further analysis.

\subsection{Immunohistochemistry}

Tumor samples were fixed in $10 \%$ formalin for 24 hours and processed for paraffin embedding. Sections $(5 \mu \mathrm{m})$ were used for H\&E and Immunohistochemistry (IHC) staining for RhoC (1:400, 10632-1-AP; Proteintech), HSP90 $\alpha$ (1:300, 13171-1-AP; Proteintech), HIF1 $\alpha$ (1:500, ab51608; Abcam), VEGF (1:300, 19003-1-AP; Proteintech). Images were acquired using an optical microscope (BX46, Olympus, Tokyo, Japan).

\subsection{8 | Tissue microarray analysis}

The bladder cancer tissue microarray (BL2081c) containing 183 samples (eight of normal bladder tissue, eight of adjacent normal bladder tissues, and 167 malignant tissues with grade I, II, or III) was purchased from Alenabio (Xi'an, China). The staining intensity values were determined by Image-Pro Plus software.

\subsection{9 | Immunofluorescence analysis of tissues}

Tumor samples were embedded in OCT compound with liquid nitrogen. Frozen sections $(5 \mu \mathrm{m})$ were used for immunofluorescence staining for CD31 (1:300, 550274; BD 
Biosciences) overnight at $4^{\circ} \mathrm{C}$. After that, coverslips were washed with PBS, and incubated with FITC-labeled secondary antibody for 1 hour. Finally tissue sections were counterstained with DAPI for nuclei visualization. Images were acquired under a fluorescence microscope (IX73, Olympus).

\subsection{0 | Statistical analyses}

The statistical significance of the differences between groups were calculated using the two-tailed Student's $t$ test, nonparametric Mann-Whitney test, or ANOVA using SPSS 22.0 software (IBM Corp., Armonk, NY, USA).

(A)

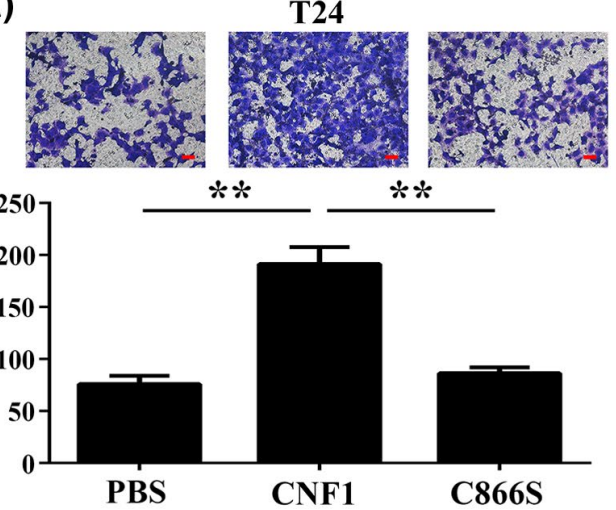

(C)

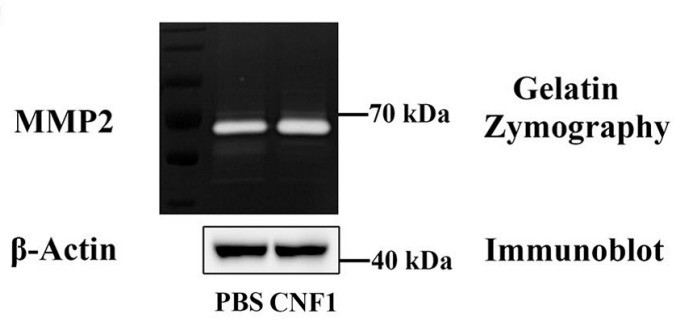

(D)
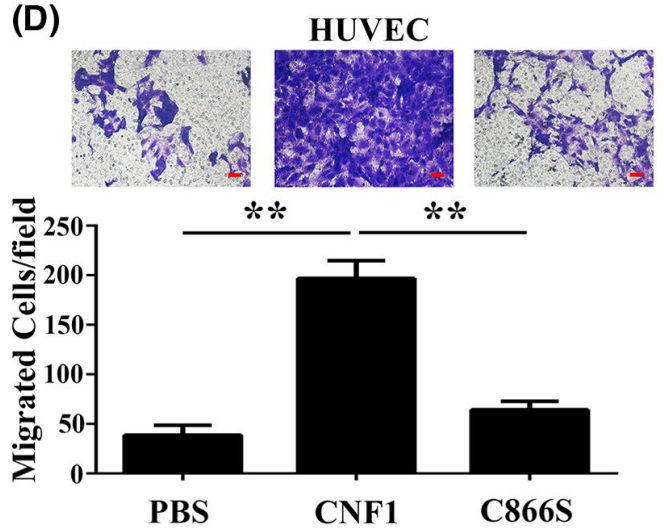

\section{3 | RESULTS}

\section{1 | CNF1 promotes the migration and invasion of bladder cancer cells and vascular endothelial cells in vitro}

We first examined the effects of CNF1 on the migration and invasion abilities of bladder cancer cells. We purified and validated CNF1 and C866S (an inactive mutant of CNF1) recombinant proteins from UPEC strain as we previously described, ${ }^{6}$ and treated bladder cancer cell lines 5637 and T24. The Transwell assay showed that wild-type CNF1 markedly enhanced the migration and invasion of T24 and 5637 cells. (Figure 1A,B, Figure S1A,B). The

(B) T24
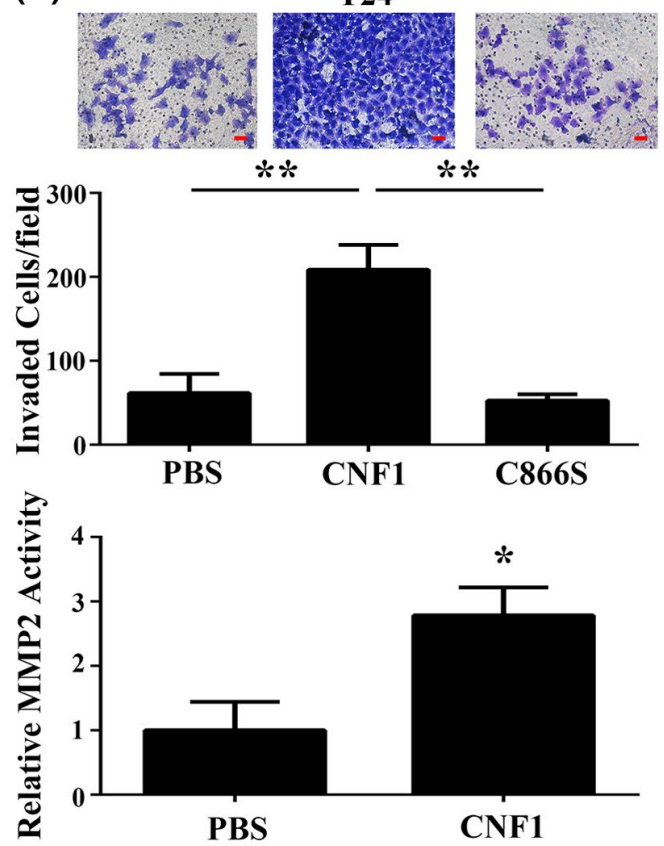

(E)

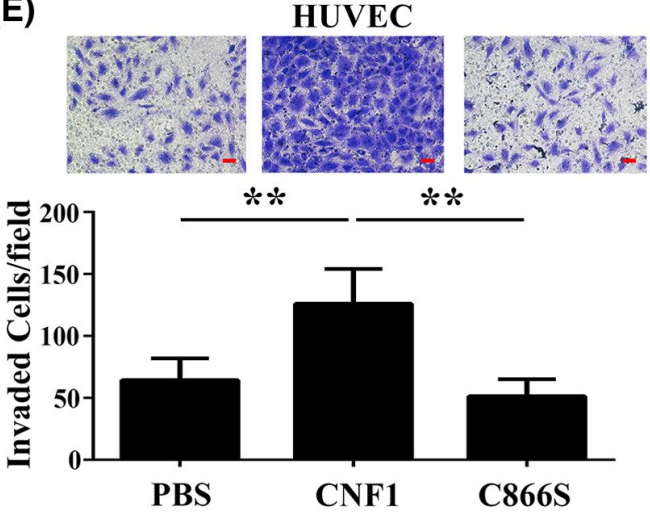

F I G U R E 1 CNF1 promotes the migration and invasion of bladder cancer cells and vascular endothelial cells. Transwell-based migration and invasion assays of T24 cells (A,B) and HUVECs (D, E) treated with $1 \mathrm{nmol} / \mathrm{L}$ recombinant CNF1 protein, PBS, and the inactivated CNF1 mutant C866S ( $\mathrm{n}=3$, three independent experiments). C, Zymography assay of MMP2 activities in 5637 cells treated with PBS and $1 \mathrm{nmol} / \mathrm{L}$ CNF1 ( $\mathrm{n}=3$, three independent experiments). $\beta$-Actin was loaded as an internal control. Data are the mean $\pm \mathrm{SD}$. $* P<.05$, **P<.01; one-way ANOVA (A, B, D, and E) or Student's $t$ test (C). Scale bar $=50 \mu \mathrm{m}$ 
gelatinases (MMP2 and MMP9) play a pivotal role in degrading the extracellular matrix (ECM). ${ }^{25}$ Accordingly, we explored whether the increased invasive effect could be attributed to MMP2 or MMP9 using a gelatin zymography assay. The results showed that MMP2 activity was increased in 5637 cells treated with CNF1 (Figure 1C), and MMP9 activity was not detected in 5637 cells. Both MMP2 and MMP9 activity were not detected in T24 cells (data not shown). We further validated the effects of CNF1 on another bladder cancer cell line (UMUC3), which indicated that CNF1 enhanced MMP9 but not MMP2 activity (Figure S1C). Taken together, these results provide strong evidence that CNF1 promotes the migration and invasion of bladder cancer cells and that MMPs may contribute to this activity.

Endothelial cells are an important component of the microenvironment; therefore, we examined the impact of CNF1 on motility and invasiveness of human umbilical vein vascular endothelial cells (HUVECs). We found CNF1 promoted migration and invasion of HUVECs (Figure 1D,E). Therefore, we speculated that CNF1 might have a synergistic effect on both the cancer cells and angiogenesis.

\subsection{CNF1 induces VEGF secretion in bladder cancer cells and subsequently promotes angiogenesis in HUVECs}

VEGF is closely associated with tumor angiogenesis, ${ }^{26}$ and tumor cells are one of the main producers of VEGF in the tumor microenvironment. In addition, tissue hypoxia is a common feature of solid tumors. ${ }^{27}$ To determine whether CNF1 could promote VEGF production from bladder cancer cells, we treated T24 or 5637 cells with CNF1, and determined VEGF secretion under normoxic and hypoxic conditions. CNF1 significantly promoted the VEGF secretion from T24 and 5637 cells under hypoxic conditions, but not under normoxic conditions (Figure 2A, Figure S2A). CNF1 promoted VEGF secretion in a time- and concentrationdependent manner in T24 and 5637 cells (Figure 2B,C, Figure S2B,C).

Angiogenesis mainly involves the endothelial cell migration and tube formation to form new blood vessels. ${ }^{28}$ Thus, we next examined whether CNF1-induced VEGF secretion stimulated angiogenesis in a HUVEC model in vitro. The results demonstrated that conditioned medium (CM) from CNF1-treated T24 and 5637 cells dramatically enhanced the tube formation of HUVECs (Figure 2D, Figure S2D). However, CNF1 did not play a direct role in promoting angiogenesis by itself (Figure S2E). These data suggest that CNF1 promotes angiogenesis by inducing the VEGF secretion in bladder cancer cells.

\section{3 | Involvement of HIF1 $\alpha$ in CNF1-induced VEGF secretion}

We next explored the mechanism through which CNF1 induces the VEGF expression. CNF1 markedly increased the VEGF mRNA level in T24 cells by qRT-PCR analysis (Figure 2E). As a pivotal transcription factor, HIF $1 \alpha$ is critical for VEGF transcription in tumor angiogenesis. ${ }^{29}$ Therefore, we investigated the possible effect of CNF1 on HIF $1 \alpha$. We treated T2 4 cells with CNF1 for $24 \mathrm{~h}$ and identified that CNF1 upregulated the HIF1 $\alpha$ protein level, but not its mRNA expression, compared with that in the control groups (Figure 2F, Figure S3A). We also ruled out the possible role of LPS in inducing HIF1 $\alpha$ (Figure 2F). We then explored the mechanism by which CNF1 increases the protein levels of HIF $1 \alpha$. The addition of the proteasome inhibitor MG132 strongly blocked CNF1-mediated HIF1 $\alpha$ upregulation under hypoxic conditions, suggesting that CNF1 promotes HIF $1 \alpha$ upregulation in a proteasome-dependent manner (Figure 2G). In addition, CNF1 effect on VEGF upregulation and increased tube formation of HUVECs were not detected in T24 and 5637 cells with HIF1A knockdown (Figure 2H-J, Figure S2F,G). These results suggest that CNF1 increases the secretion of VEGF by increasing HIF1 $\alpha$ accumulation.

\subsection{CNF1 modulates the expression of HIF1 $\alpha$ and the secretion of VEGF by activating RhoC}

Rho GTPases are the main targets of CNF1 in mammalian cells. ${ }^{30}$ To determine if Rho GTPase play a role in CNF1induced HIF1 $\alpha$ upregulation, we knocked down expression of several Rho GTPase family members (RhoA, RhoB, RhoC, Rac1, and Cdc42) using siRNAs (Figure S3B). The CNF1-mediated increase of HIF1 $\alpha$ protein levels was significantly attenuated by knockdown of RhoC compared with that achieved by siScr control, but not by knockdown of other Rho GTPases genes (Figure 3A), which suggest that CNF1 upregulates HIF1 $\alpha$ levels through RhoC. The role of RhoC in HIF1 $\alpha$ upregulation induced by CNF1 was verified on another bladder cancer cell line 5637 (Figure S3C).

We then examined the RhoC activation induced by CNF1. Treatment with CNF1 for 3 hours caused a shift in the apparent molecular mass of RhoC on SDS-PAGE (Figure 3B), indicating that the RhoC was covalently modified. We further validated the immunoblotting results using a pull-down assay (Figure 3C). These results suggest that CNF1 can upregulate the expression of HIF $1 \alpha$ by inducing the RhoC activation in bladder cancer cells. 


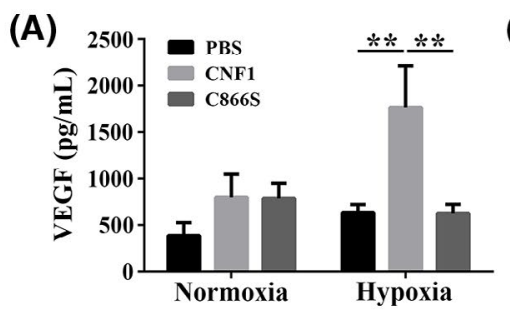

(D)
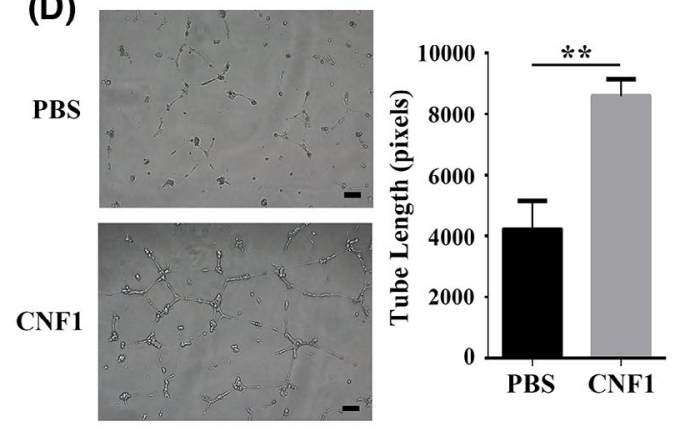

(B)

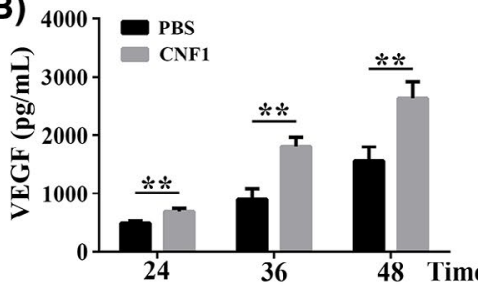

(E)

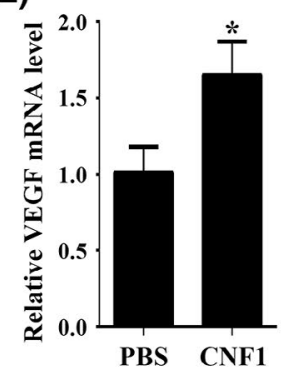

(C)

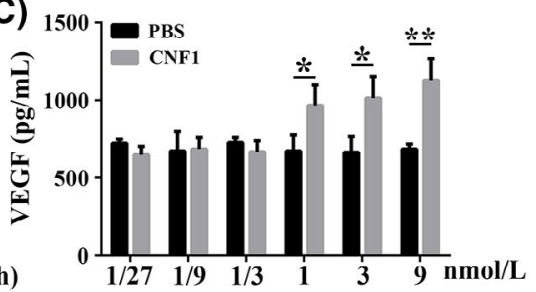

(F)

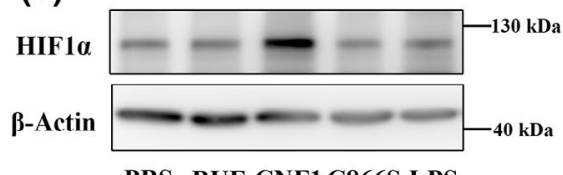

PBS BUF CNF1 C866S LPS

(H)

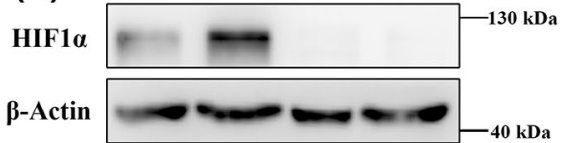

PBS CNF1 PBS CNF1

(I)

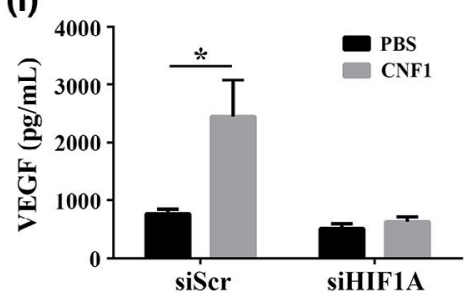

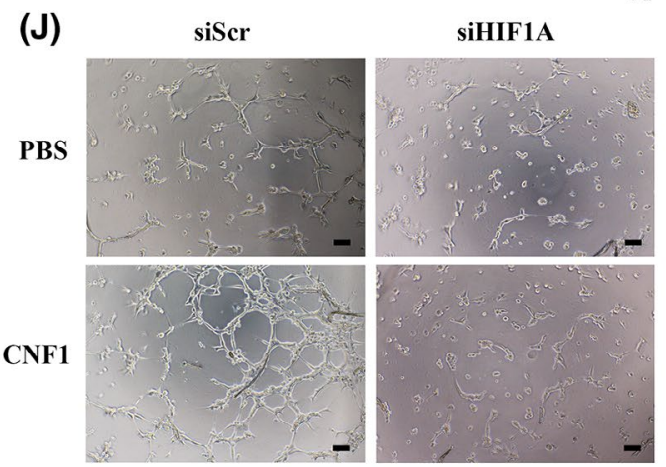

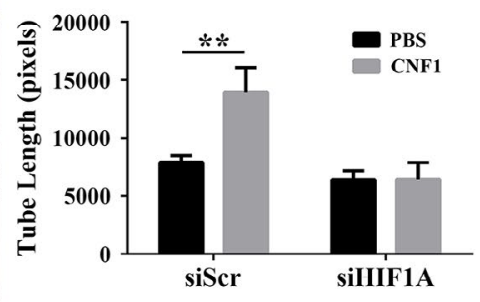

F I G U RE 2 CNF1 induces VEGF secretion in bladder cancer cells by promoting HIF1 $\alpha$ stabilization and subsequently promotes angiogenesis. A, The T24 cells were incubated with CNF1 (3 nmol/L) for 24 hours under normoxic or hypoxic conditions, and VEGF secretion in the culture medium was analyzed by ELISA $(n=3$, three independent experiments). B and C, Time- and concentration-dependent VEGF secretion in T24 cells treated by CNF1 ( $3 \mathrm{nmol} / \mathrm{L}$ ) under hypoxic conditions ( $\mathrm{n}=3$, three independent experiments). D, The T24 cells were stimulated with CNF1 ( $3 \mathrm{nmol} / \mathrm{L}$ ) or PBS for 36 hours. The medium was collected and then applied to HUVECs for 6 hours. The capillary-like structure formation in HUVECs was examined using a tube formation assay ( $\mathrm{n}=3$, three independent experiments). Tube formation ability was visualized and calculated by measuring the length of the tubes. E, T24 cells were incubated with CNF1 ( 3 nmol/L) for 24 hours under hypoxic conditions, and VEGF expression was examined by qPCR ( $n=3$, three independent experiments). F, Western blotting analysis of HIF1 $\alpha$ in T24 cells after treatment with CNF1 (3 nmol/L) under hypoxic conditions for 24 hours, with PBS, dialysis buffer, LPS $\left(1.26 \times 10^{-3} \mathrm{ng} / \mathrm{mL}\right)$, and C866S treatments as controls. G, T24 cells were pretreated with the proteasome inhibitor MG132 $(10 \mu \mathrm{mol} / \mathrm{L})$ for 1 hour followed by stimulation with CNF1 ( $3 \mathrm{nmol} / \mathrm{L}$ ) for 24 hours under normoxic or hypoxic conditions, and HIF1 $\alpha$ expression was analyzed by western blotting. H, Western blotting analysis of HIF1 $\alpha$ in $3 \mathrm{nmol} / \mathrm{L}$ CNF1-treated T24 cells transfected with scrambled or HIF1A siRNA under hypoxic conditions. I, T24 cells were transfected with scrambled or HIF1A siRNA for 24 hours followed by stimulation with CNF1 (3 nmol/L) or PBS for 36 hours. VEGF secretion in culture medium was examined by ELISA ( $\mathrm{n}=3$, three independent experiments). J, The same medium as described in (I) was applied to HUVECs for 6 hours. The capillary-like structure formation in HUVECs was examined using a tube formation assay ( $\mathrm{n}=3$, three independent experiments). Data are the mean $\pm \mathrm{SD} . * P<.05, * * P<.01$; one-way ANOVA (A-C, I and J) or Student's $t$ test (D, E). Scale bar $=100 \mu \mathrm{m}$ 


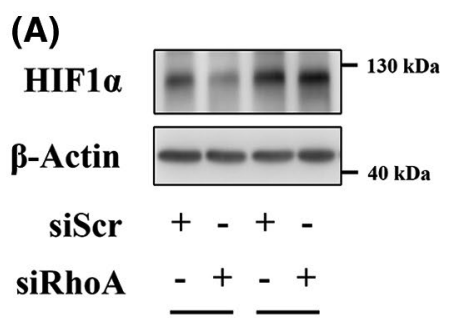

PBS CNF1

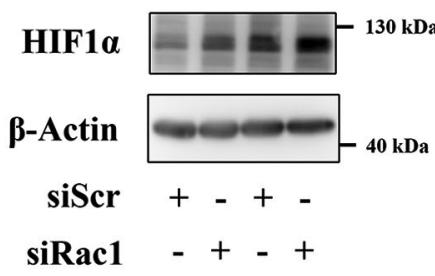

(B)


PBS CNF1

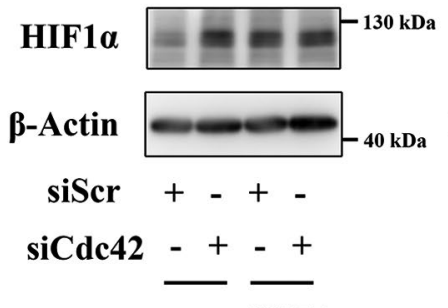

PBS CNF1
(C)

(E)

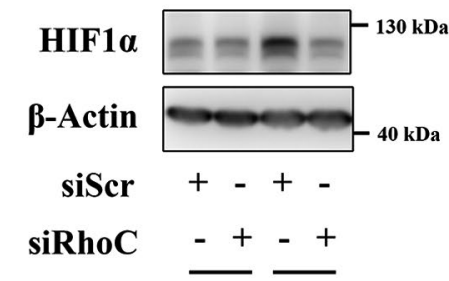

PBS CNF1
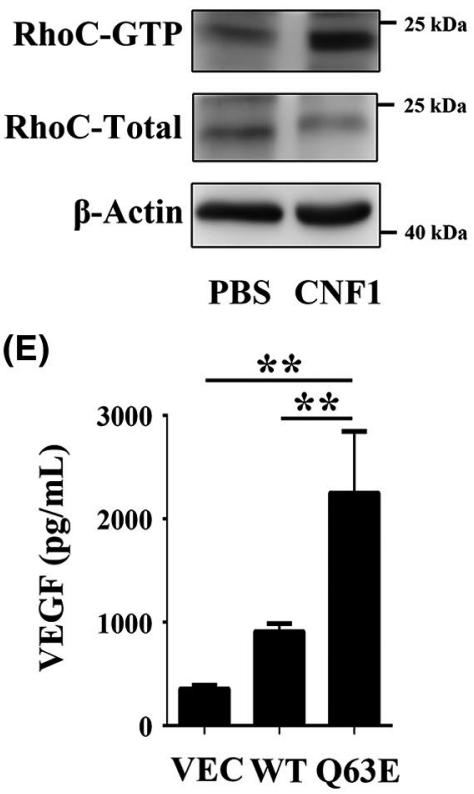

(D)

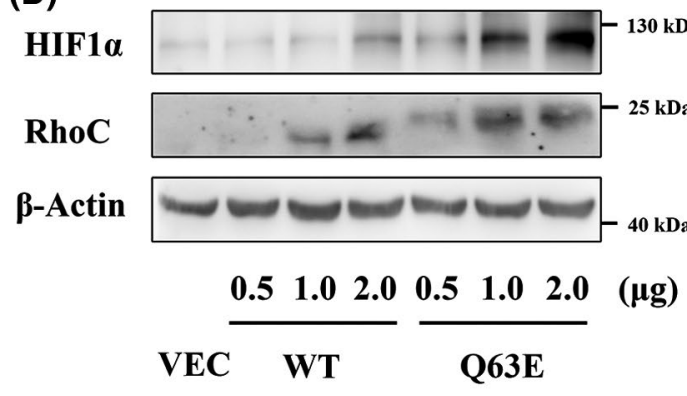

(F)

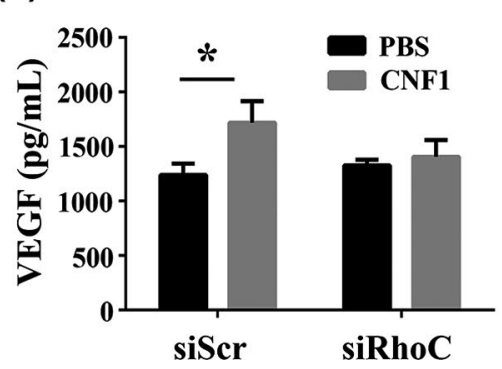

F I G U RE 3 CNF1 modulates the expression of HIF1 $\alpha$ and the secretion of VEGF by activating RhoC in bladder cancer cells. A, T24 cells were transfected with a scrambled control siRNA or those targeting respective Rho GTPase siRNAs for 24 hours, followed by stimulation with PBS or CNF1 ( $3 \mathrm{nmol} / \mathrm{L}$ ) for another 24 hours under hypoxic conditions, and HIF1 $\alpha$ expression was examined by western blotting. B, T24 cells were incubated with PBS or CNF1 for 3 to 36 hours and mobility-shifting was examined by electrophoresis. C, Western blotting analysis of activated RhoC in T24 cells treated with recombinant CNF1 protein ( $3 \mathrm{nmol} / \mathrm{L}$ ) or PBS for 24 hours after immunoprecipitation with GTP pull-down assays using anti-RhoC antibody. D, Western blotting analysis of T24 cells transfected with vector, wild-type RhoC, or constitutively active RhoC (Q63E) under hypoxic conditions. E, T24 cells were transfected with vector, wild-type RhoC, or Q63E for 48 hours, and VEGF secretion in the culture medium was examined by ELISA ( $\mathrm{n}=3$, three independent experiments). F, T24 cells were transfected with scrambled or RhoC siRNA for 24 hours followed by stimulation with CNF1 protein ( $3 \mathrm{nmol} / \mathrm{L}$ ) or PBS for 24 hours, and VEGF secretion in culture medium was examined by ELISA ( $\mathrm{n}=3$, three independent experiments). Data are the mean \pm SD. $* P<.05, * * P<.01$; one-way ANOVA (E, F)

To determine the role of active RhoC in the expression of HIF1 $\alpha$ and the secretion of VEGF, wild-type RhoC (WT-RhoC, WT) or the constitutively active RhoC mutant (CA-RhoC, Q63E; Q63 was deamidated by $\mathrm{CNF}^{7}$ ) was transfected into T24 and 293T cells. Cells transfected with CA-RhoC had higher levels of HIF1 $\alpha$ and their protein levels were significantly increased in a concentration-dependent manner compared with T24 and 293T cells transfected with vector or WT-RhoC (Figure 3D, Figure S3D). However, transfection did not significantly increase the mRNA level of HIF1 $\alpha$ (Figure S3E).

We further analyzed the possible effect of CA-RhoC on VEGF secretion. The results confirmed that overexpression of CA-RhoC effectively increased the secretion of VEGF 
compared with that in vector or WT-RhoC groups in T24 and 5637 cells (Figure 3E, Figure S3F). In addition, transfection with RhoC siRNA inhibited the CNF1-enhanced secretion of VEGF (Figure 3F).

Taken together, these results indicate that CNF1 enhances HIF1 $\alpha$ stabilization and VEGF secretion by activating RhoC in bladder cancer cells.

\section{5 | CNF1-induced RhoC activation modulates the stabilization of HIF1 $\alpha$ by upregulating HSF1-HSP90a}

To explore how active RhoC promotes the stabilization of HIF1 $\alpha$, we compared the transcriptomes of T24 cells with CA-RhoC overexpression or vector control by RNA-Seq. We analyzed the genes associated with HIF1 $\alpha$ degradation under hypoxic conditions (Figure 4A). To verify some of the relevant upregulated genes in the RNA-seq data, qRT-PCR was performed (Figure 4B). Among these genes, we found that the mRNA level of HSP90AA1 was significantly upregulated in CA-RhoC overexpressing cells. Western blotting analysis also revealed an increased protein level of HSP90 $\alpha$ in CARhoC overexpressing T24 cells (Figure 4C). We further validated the effects of CNF1 on HSP90 $\alpha$, which revealed that CNF1 also increased HSP90 $\alpha$ expression (Figure 4D). HSP90 can directly interact with HIF1 $\alpha$ and promotes HIF $1 \alpha$ stabilization under hypoxic conditions, ${ }^{22}$ which led us to examine whether HSP90 $\alpha$ is involved in RhoC-facilitated HIF1 $\alpha$ stabilization. We transfected T24 or 293T cells with HA-tagged HSP90 $\alpha$, MYC-tagged HIF1 $\alpha$ and FLAG-tagged CA-RhoC or vector. Co-IP analysis indicated that CA-RhoC enhanced the interaction between HSP90 $\alpha$ and HIF1 $\alpha$ under hypoxic conditions (Figure 4E, Figure S4A).

$\mathrm{NF}-\kappa \mathrm{B}$ and HSF1 were reported to regulate the expression of HSP90 $\alpha .{ }^{31}$ Ser326 phosphorylation is important for the transcriptional activity of HSF1. ${ }^{31,32}$ We found increased levels of HSF1 phosphorylated at Ser326, HSP90 $\alpha$, and HIF1 $\alpha$ after CA-RhoC overexpression (Figure 4F,G) or CNF1 treatment (Figure $4 \mathrm{H}$ ), but there was no change in NF- $\kappa \mathrm{B}$ activity (Figure S4B). We blocked HSF1 and NF- $\kappa B$ using commercial inhibitors (Figure 4F, Figure S4C) and found that enhanced levels of phosphorylated HSF1, HSP90 $\alpha$, and HIF1 $\alpha$ were attenuated by inhibiting HSF1, but not by inhibiting NF- $\mathrm{KB}$. To further confirm the role of HSF1 in promoting HSP90 $\alpha$ and HIF1 $\alpha$ levels, we knocked down HSF1 using specific siRNAs, and found that CARhoC or CNF1 induced HSF1 phosphorylation, HSP90 $\alpha$ and HIF $1 \alpha$ increasement were not observed (Figure 4G,H, Figure S4D).The effect of HSF1 on HIF1 $\alpha$ in 5637 cells was also validated (Figure S4E). CNF1 effect on VEGF upregulation was not detected in T24 and 5637 cells with HSF1 knockdown (Figure 4I, Figure S4F).
The above results demonstrate that CNF1-induced RhoC activation can increase the HIF1 $\alpha$ stabilization in bladder cancer cells through the HSF1-HSP90 $\alpha$ axis.

\subsection{Active RhoC promotes in vivo tumor- associated angiogenesis of bladder cancer}

To determine if the in vitro observations could be replicated in vivo, the effect of RhoC activation on the angiogenesis mediated by the bladder cancer cells was analyzed in vivo using a subcutaneous tumor mouse model. T24 cells were transduced with constitutively expressed CA-RhoC or the vector control. The expression of RhoC was confirmed in the transduced cells, and elevated HSP90 $\alpha$ and HIF1 $\alpha$ levels were also identified in T24 cells with CA-RhoC overexpression (Figure S4G). Five weeks after transplantation, we observed significantly the elevated tumor growth in the CA-RhoC overexpression group compared with that in the control group (Figure 5A,B). The tumor volume and weight were 3.6- and 2.4-fold higher than those in the control group (Figure 5C,D). In addition, tumors in mice receiving the T24 cells with CA-RhoC had higher levels of HSP90 $\alpha$, HIF1 $\alpha$, and VEGF (Figure 5E-H). We also found increased microvessel density (MVD) in tumors derived from the CA-RhoCoverexpressing T24 cells compared with those in the control group (Figure 5I).

Overall, these results suggest that activation of RhoC by $\mathrm{CNF} 1$ promotes angiogenesis and tumor growth in vivo.

\section{7 | HSP90 $\alpha$, HIF1 $\alpha$, and VEGF are implicated in advanced human bladder cancer}

To identify the possible clinical correlations to our findings, the expression profiles of HSP90 $\alpha$, HIF1 $\alpha$, and VEGF were analyzed by IHC staining using human tissue arrays containing 183 samples including 167 bladder carcinoma tissues of grade I, II, or III, eight normal and eight adjacent normal bladder tissues. We found that the levels of HSP90 $\alpha$, HIF $1 \alpha$, and VEGF were strongly correlated with bladder cancer advanced grades (Figure 6A-D). Furthermore, the levels of their expression were correlated with each other (Figure 6E-G). In addition, we performed the analysis using the integrated cancer microarray database, Oncomine, ${ }^{33}$ which revealed that HSP90AA1 (Figure 6H) and HIF1A (Figure 6I) mRNA levels are markedly upregulated in bladder cancer samples. We also analyzed the dataset from The Cancer Genome Atlas (TCGA). ${ }^{34}$ The HSP90AA1 mRNA level (based on 407 bladder cancer samples) was significantly higher compared with the adjacent normal bladder tissues of 18 bladder cancer samples, and no difference was observed for HIF1A (Figure 6J,K). However, both HSP90AA1 and HIF1A mRNA levels were 
(A)

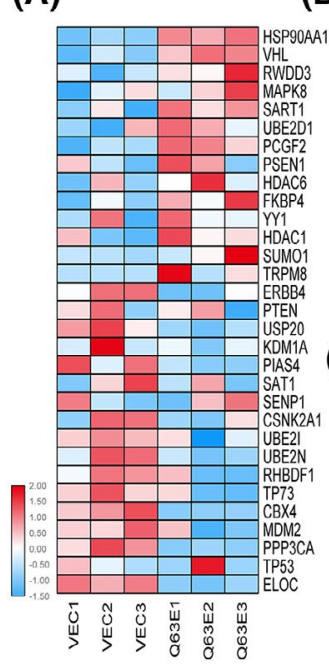

(F)

$$
(\mathbf{F )}
$$

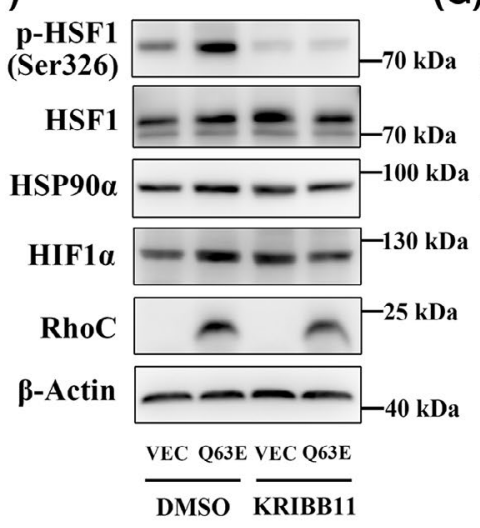

(B)

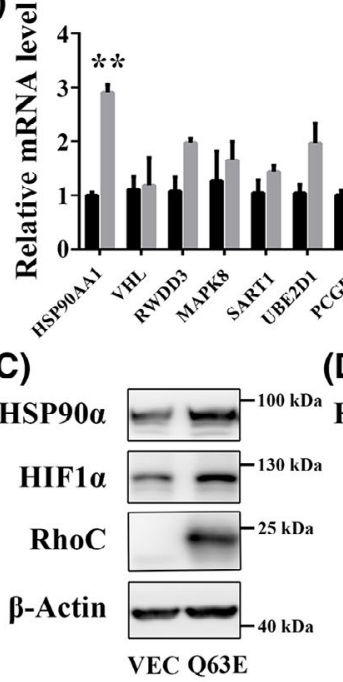

(G)
(D)

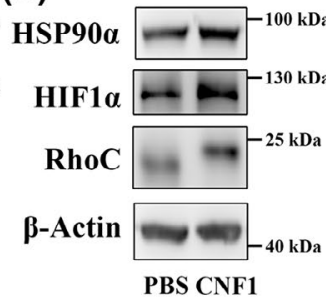

(E)

VEC Q63E MYC-HIF1a ++++

HA-HSP90ג ++++ FLAG-RhoC-Q63E - + - +

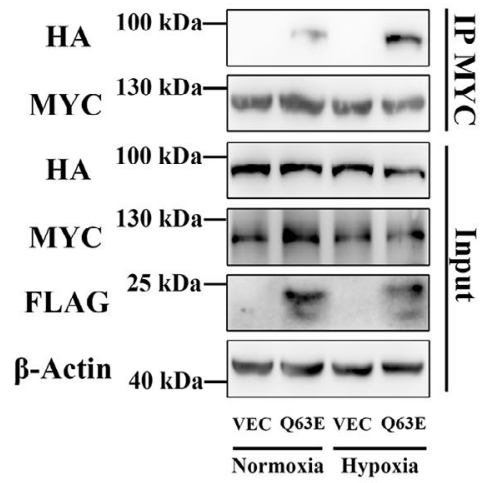

(H)

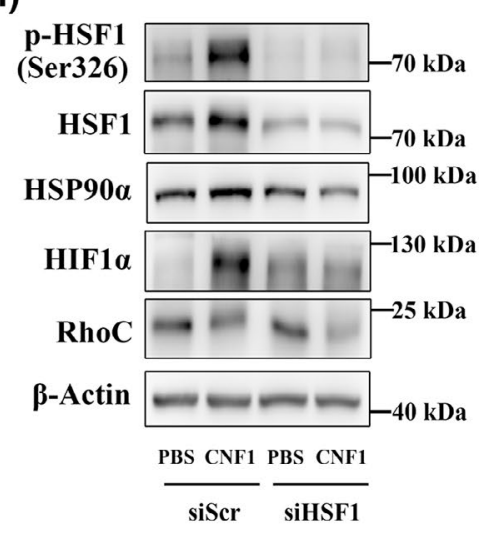

(I)

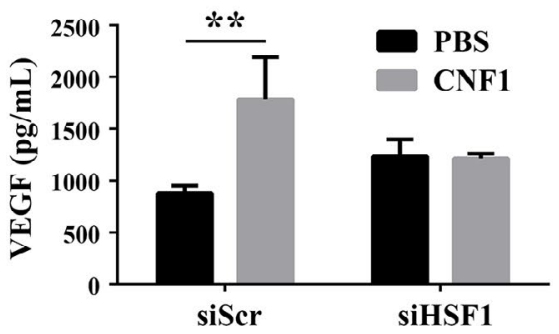

F I G U RE 4 CNF1-induced RhoC activation modulates the HIF1 $\alpha$ stabilization by upregulating HSF1- HSP90 $\alpha$ interaction. A, Heatmap of the HIF1 $\alpha$ degradation-related gene expression levels detected by RNA-seq in T24 cells stably expressing the RhoC constitutively active mutant Q63E or vector under hypoxic condition. B, qRT-PCR confirmation of the upregulated genes expression profile under hypoxic condition $(\mathrm{n}=3$, three independent experiments). C, Western blotting analysis of T24 cells transduced with vector and Q63E under hypoxic condition. D, Western blotting analysis of T24 cells treated with $1 \mathrm{nmol} / \mathrm{L} \mathrm{CNF1.} \mathrm{E,} \mathrm{T24} \mathrm{cells} \mathrm{were} \mathrm{transfected} \mathrm{with} \mathrm{combinations} \mathrm{of} \mathrm{MYC-HIF1} \alpha$, HA-HSP90 $\alpha$, and FLAG-RhoC-Q63E under normoxic or hypoxic condition. Protein extracts from the transfected cells were subjected to IP with antibody against MYC and analyzed by immunoblotting with the indicated antibodies. F, Analysis of phosphorylated HSF1, total HSF1, HSP90 $\alpha$, and HIF1 $\alpha$ in transduced T24 cells treated with the HSF1 inhibitor KRIBB11 $(10 \mu \mathrm{mol} / \mathrm{L})$, or with DMSO as the control under hypoxic condition. G and $\mathrm{H}$, Western blotting analysis of transduced T24 cells $(\mathrm{G})$ and $1 \mathrm{nmol} / \mathrm{L}$ CNF1 treated T24 cells $(\mathrm{H})$ subject to siRNAs targeting HSF1 and scrambled non-targeting control siRNA under hypoxic conditions. I, T24 cells were transfected with scrambled or HSF1 siRNA for 24 hours followed by stimulation with CNF1 ( $3 \mathrm{nmol} / \mathrm{L}$ ) or PBS for 24 hours under hypoxic condition, and VEGF secretion in culture medium was examined by ELISA ( $\mathrm{n}=3$, three independent experiments). Data are the mean $\pm \mathrm{SD}$. $* * P<.01$; one-way ANOVA (B, I)

found significantly increased in 18 bladder cancer samples compared with their paired adjacent normal bladder tissues (Figure 6L,M). Furthermore, we performed the Kaplan-Meier survival analysis from the Gene Expression Profiling Interactive Analysis (GEPIA) web server. ${ }^{35}$ Elevated HIF1A expression is positively correlated with the poor survival in 
(A)

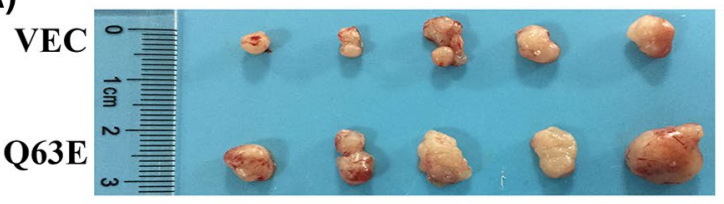

(B)

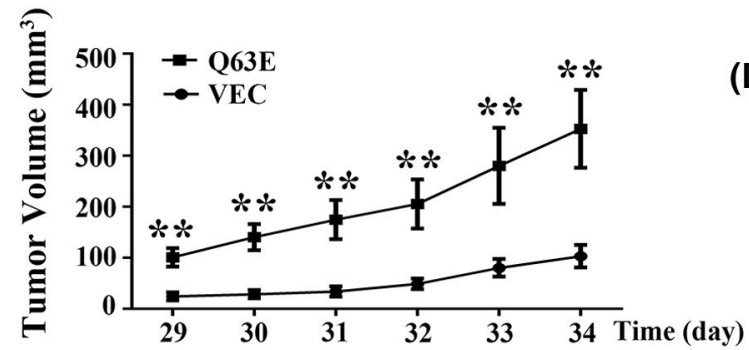

(C)

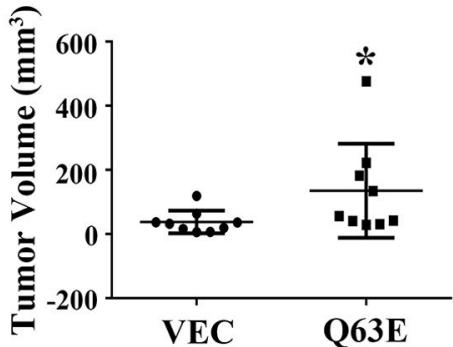

(D)

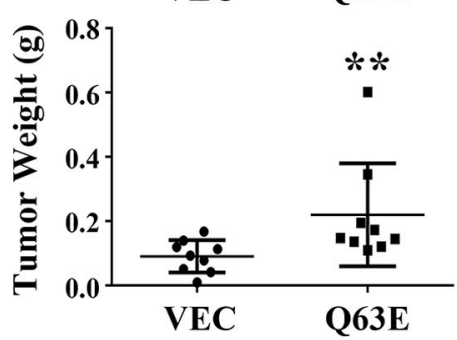

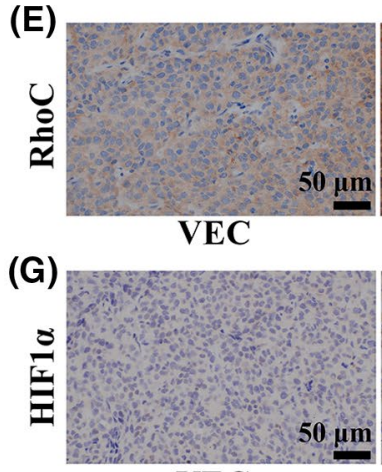

VEC

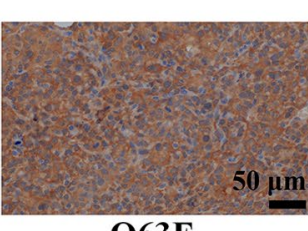

Q63E

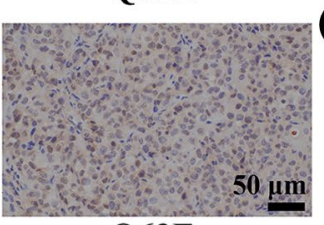

Q63E
(F)

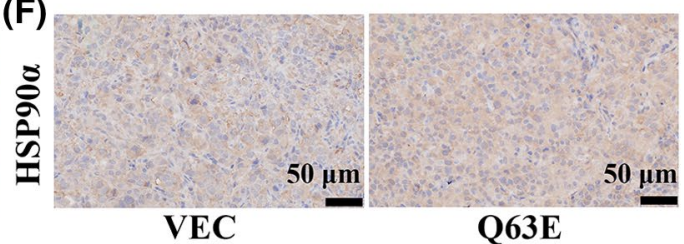

(I)

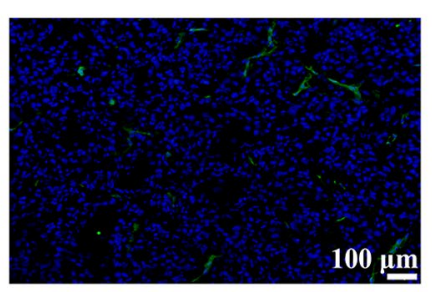

VEC

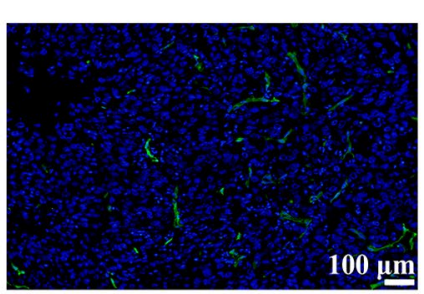

Q63E

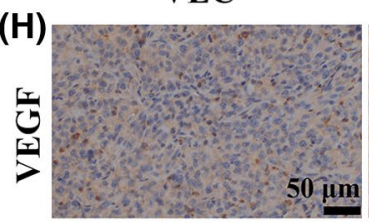

VEC

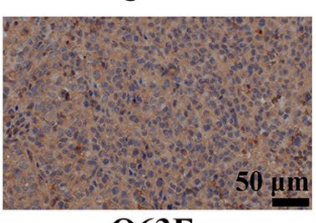

Q63E

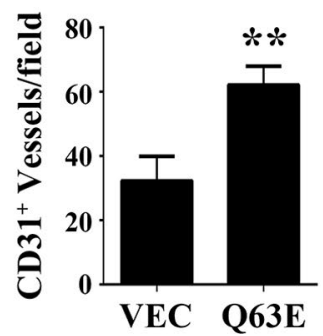

F I G U R E 5 Active RhoC promotes the tumor-associated angiogenesis of bladder cancer in vivo. A, Morphological images of tumor xenografts resected from nude mice injected with T24 cells transduced with vector (VEC) or constitutively active mutant of RhoC (Q63E) after 5 weeks in each group. B, Tumor growth in nude mice with Q63E or VEC T24 cells subcutaneously injected into their flanks ( $\mathrm{n}=9$, two independent experiments). Tumor volumes were determined by direct measurement using a caliper and calculated using the formula: (widest diameter $\times$ smallest diameter $\left.{ }^{2}\right) / 2$. Tumor volume (C) and weight (D) of xenograft nude mice injected with Q63E or VEC T24 cells in the xenograft model ( $\mathrm{n}=9$, two independent experiments). E-H, Immunohistochemical analysis of RhoC, HSP90 $\alpha$, HIF1 $\alpha$, or VEGF expression in murine tumors. I, Immunofluorescence analysis of $\mathrm{CD} 31^{+}$blood vessels in murine tumors $(\mathrm{n}=3$, three independent experiments each with multiple fields). Data are the mean $\pm \mathrm{SD} . * P<.05, * * P<.01$; non-parametric Mann-Whitney test $(\mathrm{B}, \mathrm{C}, \mathrm{D}$ and I). Scale bar $=50 \mu \mathrm{m}(\mathrm{E}-\mathrm{H})$ or $100 \mu \mathrm{m}(\mathrm{I})$

patients with bladder carcinoma, and a trend without statistical significance was observed for HSP90AA1 (Figure 6N,O). We also analyzed published data from the GEO database. ${ }^{36}$ Analysis of two published clinical datasets (GSE83586 and GSE101723) revealed significantly positive correlations between the expression of HSP90AA1 and HIF1A in bladder cancer (Figure 6P). Taken together, these observations support that the HSP $90 \alpha$-HIF $1 \alpha$-VEGF axis plays an important role in the bladder cancer development.

\section{DISCUSSION}

CNF1 has been reported to activate several Rho GTPases by deamidating specific glutamine residues. ${ }^{7}$ CNF1-mediated Rho GTPase activation changes the cell function and contributes to many physiological and pathological processes. For example, CNF1 promotes bacterial invasion into host cells by activating Rac. ${ }^{37}$ Previously, we reported that CNF1 increased prostate cancer cell migration and invasion 
(A)

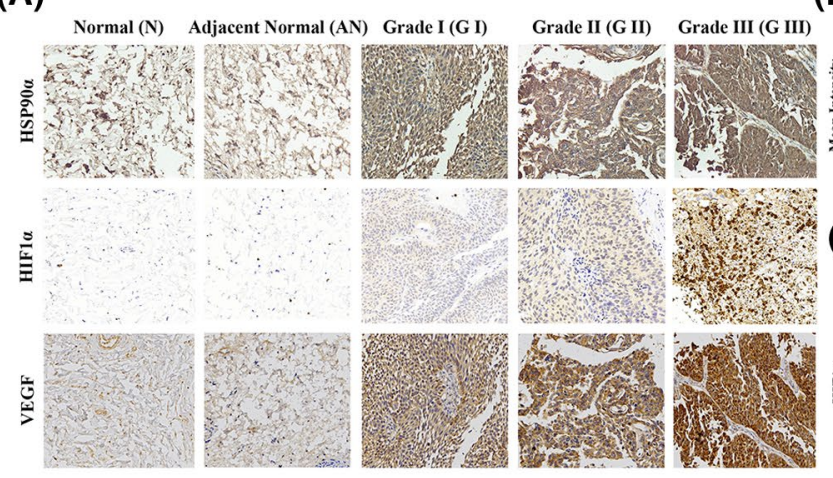

(B)

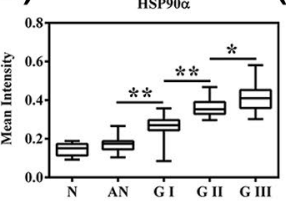

(E)

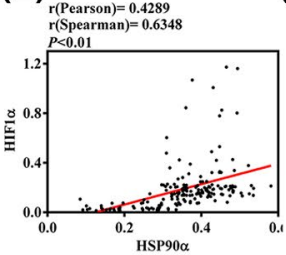

(C)

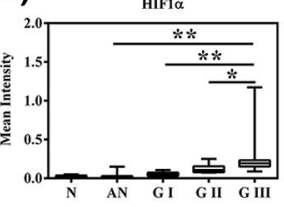

(F)

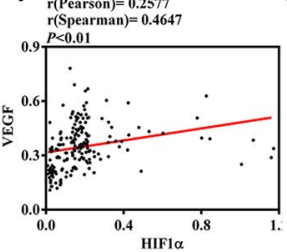

(D)

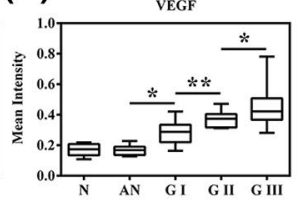

(G)

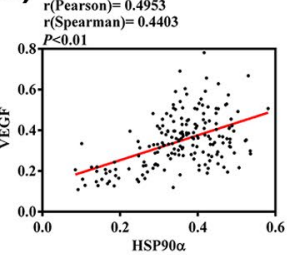

(H)

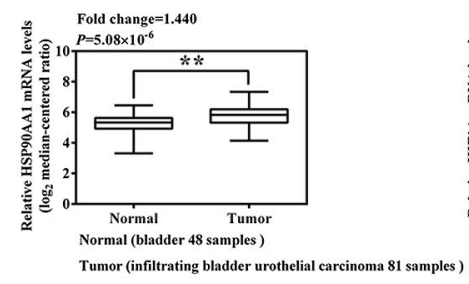

(I)

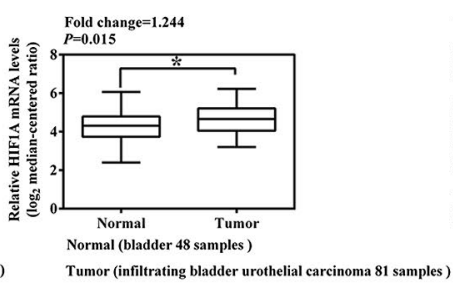

(J)

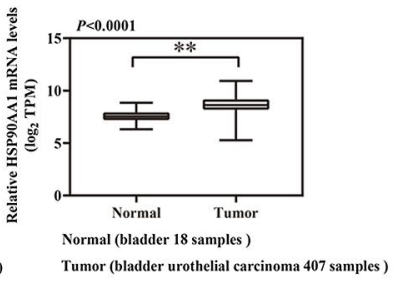

(K)
(L)

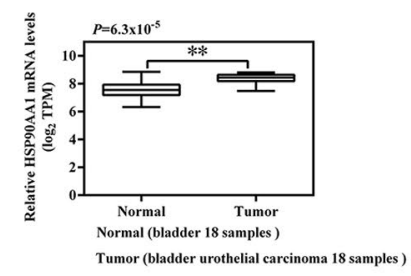

(M)

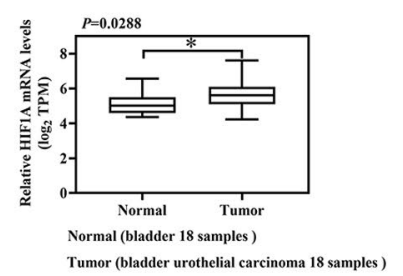

(N)

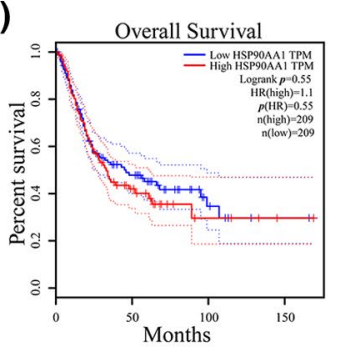

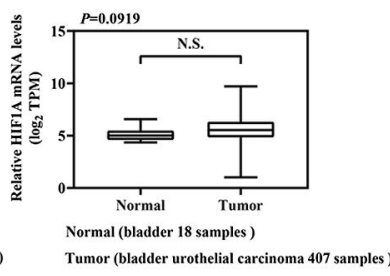

(0)

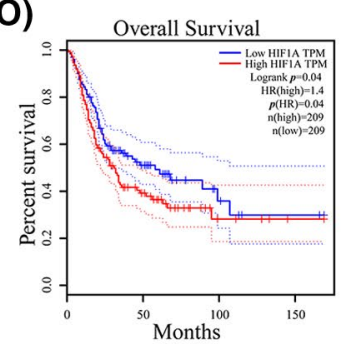

(P)
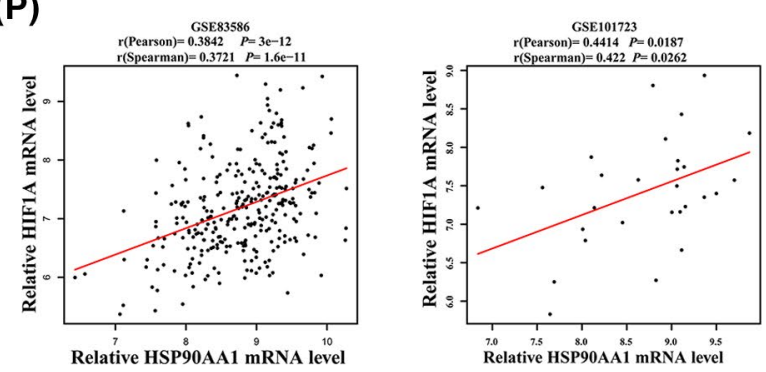

F I G U RE 6 HSP90 $\alpha$, HIF $1 \alpha$, and VEGF expressions are positively correlated with advanced human bladder cancer. A, IHC analysis of HSP90 $\alpha$, HIF1 $\alpha$, and VEGF using human bladder cancer tissue microarray. Representative images (original magnification 200×) from normal bladder tissues, adjacent normal bladder tissues, and malignant bladder tissues at different stages are shown. B-D, Mean intensity of staining of HSP90 $\alpha$ (B), HIF1 $\alpha$ (C), and VEGF (D) were determined by Image-Pro Plus software and presented with box plots. E-G, The correlation coefficient and $P$ values were analyzed as indicated. $\mathrm{H}$ and I, Analysis of Sanchez-Carbayo bladder from Oncomine for the expression of HSP90AA1 (H) and HIF1A (I) in normal human bladder tissues and bladder carcinoma samples. J and K, Analysis of the mRNA level of HSP90AA1 (J) and HIF1A (K) in 407 bladder cancer and 18 adjacent normal bladder tissues obtained from the TCGA database. L and M, Analysis of TCGA data set for the mRNA expression of HSP90AA1 (L) and HIF1A (M) in 18 bladder cancer samples and the paired adjacent normal bladder tissues. $\mathrm{N}$ and $\mathrm{O}$, Kaplan-Meier survival analysis for the relationship between the survival of bladder cancer patients and expression levels of HSP90AA1 (N) and HIF1A (O) mRNA using the online tool (http://gepia.cancer-pku.cn/). P, Analysis of public datasets (GSE83586, GSE101723) for the expression of HIF1A and HSP90AA1 in bladder cancer. The relative levels of HSP90AA1 were plotted against that of HIF1A. Data are presented with box plots. ${ }^{*} P<.05$, ${ }^{*} P<.01$; one-way ANOVA (B-D); Pearson's correlation coefficient and Spearman's correlation coefficient (E-G, P); unpaired, two-tailed Student's t test (H-K); paired, two-tailed Student's $t$ test (L, M) or logrank test (N, O) 
to promote prostate cancer metastasis through activating Cdc $42,{ }^{6}$ and $\mathrm{CNF} 1$ reduced macrophage phagocytosis to induce inflammation during acute UTIs partially through Cdc42. ${ }^{38}$ Schmidt reported that CNF1 enhanced breast cancer cell invasion through activating RhoC. ${ }^{39}$

RhoC has been reported to play important roles in tumor progression, including angiogenesis, ${ }^{40-42}$ proliferation, ${ }^{43}$ invasion, and metastasis of tumors. ${ }^{11,44} \mathrm{RhoC}$ has been regarded as a new target for therapeutic vaccination against metastatic cancer. ${ }^{45}$ The mRNA and protein levels of RhoC are significantly higher in bladder cancer than in normal tissue. ${ }^{46}$ For bladder cancer, previous studies showed that RhoC was involved in its lung colonization, ${ }^{47}$ and the RhoC/ROCK pathway was closely associated with its invasion and metastasis. ${ }^{48}$ In addition, RhoC is correlated with the angiogenic component FGF2 in urothelial cell carcinoma of the bladder. ${ }^{49}$

$\mathrm{RhoC}$ was shown to induce angiogenesis by regulating endothelial cell migration and organization, ${ }^{41}$ and to maintain vascular homeostasis by modulating the endothelial cell proliferation and permeability. ${ }^{42}$ In addition, reports have shown that RhoC increases the VEGF expression. ${ }^{40,50-52}$ It was reported that $\mathrm{p} 38$ is involved in RhoC-induced VEGF production in breast cancer cells. ${ }^{52}$ However, the detailed specific mechanism through which RhoC accelerates the VEGF secretion in bladder cancer remains unclear.

HIF1 $\alpha$ stabilization is important for VEGF transcription, and several mechanisms, in addition to the classical pVHL pathway, have been reported to stabilize HIF1 $\alpha$ : (1) HSP90 could directly interact with HIF $1 \alpha$ and protect it from degradation $^{22}$; (2) increased expression of small ubiquitin-like modifier SUMO-1 under hypoxic conditions results in HIF1 $\alpha$ SUMOylation and stabilization ${ }^{53,54}$; (3) USP20, a deubiquitinase, binds to HIF1 $\alpha$ and subsequently removes ubiquitin from HIF $1 \alpha$, leading to its stabilization ${ }^{55}$; and (4) YY1, a transcription factor, can directly bind to and stabilizing HIF $1 \alpha .{ }^{56}$ In the present study, we examined the transcription of factors associated with HIF1 $\alpha$ stabilization and identified that activated RhoC induced HIF $1 \alpha$ stabilization and VEGF production by increasing the HSP90 $\alpha$ expression and the interaction between HIF1 $\alpha$ and HSP90.

\section{ACKNOWLEDGMENTS}

This study was supported by grants from the National Natural Science Foundation of China (NSFC) Programs (31970133, 81672740, 81572882, 31670071), the National Key Technologies R\&D Program, Intergovernmental international innovation cooperation (2018YFE0102000), the Science \& Technology Development Fund of Tianjin Education Commission for Higher Education (2017ZD12), Tianjin Science and Technology Commissioner Project (18JCZDJC36000).

\section{CONFLICT OF INTEREST}

The authors declare no conflict of interest.

\section{AUTHOR CONTRIBUTIONS}

Q. Wang, Z. Yao, and Z.-S. Zhang designed the study; Y. Guo, J. Wang, K. Zhou, J. Lv, L. Wang, and S. Gao performed the majority of experiments; Q. Wang, Z. Yao, Y. Guo, Z.-S. Zhang, and ET Keller analyzed the data and wrote the paper. All authors discussed the data, and reviewed the manuscript.

\section{REFERENCES}

1. Bien J, Sokolova O, Bozko P. Role of uropathogenic Escherichia coli virulence factors in development of urinary tract infection and kidney damage. Int J Nephrol. 2012;2012:681473.

2. Nielubowicz GR, Mobley HL. Host-pathogen interactions in urinary tract infection. Nat Rev Urol. 2010;7:430-441.

3. Mitsumori K, Terai A, Yamamoto S, Ishitoya S, Yoshida O. Virulence characteristics of Escherichia coli in acute bacterial prostatitis. J Infect Dis. 1999;180:1378-1381.

4. Tolg C, Sabha N, Cortese R, et al. Uropathogenic E coli infection provokes epigenetic downregulation of CDKN2A (p16INK4A) in uroepithelial cells. Lab Invest. 2011;91:825-836.

5. Simons BW, Durham NM, Bruno TC, et al. A human prostatic bacterial isolate alters the prostatic microenvironment and accelerates prostate cancer progression. J Pathol. 2015;235:478-489.

6. Guo Y, Zhang Z, Wei H, et al. Cytotoxic necrotizing factor 1 promotes prostate cancer progression through activating the $\mathrm{Cdc} 42$ PAK1 axis. J Pathol. 2017;243:208-219.

7. Schmidt G, Sehr P, Wilm M, Selzer J, Mann M, Aktories K. Gln 63 of Rho is deamidated by Escherichia coli cytotoxic necrotizing factor-1. Nature. 1997;387:725-729.

8. Vega FM, Ridley AJ. Rho GTPases in cancer cell biology. FEBS Lett. 2008;582:2093-2101.

9. Gomez del Pulgar T, Benitah SA, Valeron PF, Espina C, Lacal JC. Rho GTPase expression in tumourigenesis: evidence for a significant link. BioEssays. 2005;27:602-613.

10. Karlsson R, Pedersen ED, Wang Z, Brakebusch C. Rho GTPase function in tumorigenesis. Biochim Biophys Acta. 2009;1796:91-98.

11. Clark EA, Golub TR, Lander ES, Hynes RO. Genomic analysis of metastasis reveals an essential role for RhoC. Nature. 2000;406:532-535

12. Golen KL, Wu ZF, Qiao XT, Bao L, Merajver SD. RhoC GTPase overexpression modulates induction of angiogenic factors in breast cells. Neoplasia. 2000;2:418-425.

13. Yang J, Wang Q, Qiao C, et al. Potent anti-angiogenesis and anti-tumor activity of a novel human anti-VEGF antibody, MIL60. Cell Mol Immunol. 2014;11:285-293.

14. Folkman J. Tumor angiogenesis: therapeutic implications. N Engl J Med. 1971;285:1182-1186.

15. Ferrara N, Gerber HP, LeCouter J. The biology of VEGF and its receptors. Nat Med. 2003;9:669-676.

16. Semenza GL. Hypoxia, clonal selection, and the role of HIF-1 in tumor progression. Crit Rev Biochem Mol Biol. 2000;35:71-103.

17. Semenza GL. HIF-1: mediator of physiological and pathophysiological responses to hypoxia. J Appl Physiol (1985). 2000;88:1474-1480.

18. Semenza GL. Targeting HIF-1 for cancer therapy. Nat Rev Cancer. 2003;3:721-732.

19. Yu F, White SB, Zhao Q, Lee FS. HIF-1alpha binding to VHL is regulated by stimulus-sensitive proline hydroxylation. Proc Natl Acad Sci U S A. 2001;98:9630-9635. 
20. Tanimoto K, Makino Y, Pereira T, Poellinger L. Mechanism of regulation of the hypoxia-inducible factor-1 alpha by the von HippelLindau tumor suppressor protein. EMBO J. 2000;19:4298-4309.

21. Epstein AC, Gleadle JM, McNeill LA, et al. C. elegans EGL-9 and mammalian homologs define a family of dioxygenases that regulate HIF by prolyl hydroxylation. Cell. 2001;107:43-54.

22. Jochmanova I, Yang C, Zhuang Z, Pacak K. Hypoxia-inducible factor signaling in pheochromocytoma: turning the rudder in the right direction. J Natl Cancer Inst. 2013;105:1270-1283.

23. Yee Koh M, Spivak-Kroizman TR, Powis G. HIF-1 regulation: not so easy come, easy go. Trends Biochem Sci. 2008;33:526-534.

24. Shen KH, Li CF, Chien LH, et al. Role of galectin-1 in urinary bladder urothelial carcinoma cell invasion through the JNK pathway. Cancer Sci. 2016;107:1390-1398.

25. Cathcart J, Pulkoski-Gross A, Cao J. Targeting matrix metalloproteinases in cancer: bringing new life to old ideas. Genes Dis. 2015;2:26-34

26. Matsumoto K, Ema M. Roles of VEGF-A signalling in development, regeneration, and tumours. J Biochem. 2014;156:1-10.

27. Majmundar AJ, Wong WJ, Simon MC. Hypoxia-inducible factors and the response to hypoxic stress. Mol Cell. 2010;40:294-309.

28. Carmeliet P, Jain RK. Angiogenesis in cancer and other diseases. Nature. 2000;407:249-257.

29. Semenza GL. HIF-1 and tumor progression: pathophysiology and therapeutics. Trends Mol Med. 2002;8:S62-S67.

30. Fabbri A, Travaglione S, Fiorentini C. Escherichia coli cytotoxic necrotizing factor 1 (CNF1): toxin biology, in vivo applications and therapeutic potential. Toxins. 2010;2:283-296.

31. Prodromou C. Mechanisms of Hsp90 regulation. Biochem J. 2016;473:2439-2452.

32. Boellmann F, Guettouche T, Guo Y, Fenna M, Mnayer L, Voellmy R. DAXX interacts with heat shock factor 1 during stress activation and enhances its transcriptional activity. Proc Natl Acad Sci U S A. 2004;101:4100-4105.

33. Rhodes DR, Kalyana-Sundaram S, Mahavisno V, et al. Oncomine 3.0: genes, pathways, and networks in a collection of 18,000 cancer gene expression profiles. Neoplasia. 2007;9:166-180.

34. Lee J-S. Exploring cancer genomic data from the cancer genome atlas project. BMB Rep. 2016;49:607-611.

35. Tang Z, Li C, Kang B, Gao G, Li C, Zhang Z. GEPIA: a web server for cancer and normal gene expression profiling and interactive analyses. Nucleic Acids Res. 2017;45:W98-w102.

36. Clough E, Barrett T. The gene expression omnibus database. Methods Mol Biol. 2016;1418:93-110.

37. Doye A, Mettouchi A, Bossis G, et al. CNF1 exploits the ubiquitin-proteasome machinery to restrict Rho GTPase activation for bacterial host cell invasion. Cell. 2002;111:553-564.

38. Yang $\mathrm{H}$, Li Q, Wang C, et al. Cytotoxic necrotizing factor 1 downregulates CD36 transcription in macrophages to induce inflammation during acute urinary tract infections. Front Immunol. 2018;9:1987.

39. Lang S, Busch H, Boerries M, et al. Specific role of RhoC in tumor invasion and metastasis. Oncotarget. 2017;8:87364-87378.

40. Zhao ZH, Tian Y, Yang JP, Zhou J, Chen KS. RhoC, vascular endothelial growth factor and microvascular density in esophageal squamous cell carcinoma. World J Gastroenterol. 2015;21:905-912.

41. Wang W, Wu F, Fang F, Tao Y, Yang L. RhoC is essential for angiogenesis induced by hepatocellular carcinoma cells via regulation of endothelial cell organization. Cancer Sci. 2008;99:2012-2018.
42. Hoeppner LH, Sinha S, Wang Y, et al. RhoC maintains vascular homeostasis by regulating VEGF-induced signaling in endothelial cells. J Cell Sci. 2015;128:3556-3568.

43. Wu Y, Tao Y, Chen Y, Xu W. RhoC regulates the proliferation of gastric cancer cells through interaction with IQGAP1. PLOS ONE. 2012;7:e48917.

44. Liu N, Zhang G, Bi F, et al. RhoC is essential for the metastasis of gastric cancer. J Mol Med. 2007;85:1149-1156.

45. Wenandy L, Sorensen RB, Svane IM, Thor Straten P, Andersen MH. RhoC a new target for therapeutic vaccination against metastatic cancer. Cancer Immunol. Immunother. 2008;57:1871-1878.

46. Volanis D, Zaravinos A, Kadiyska T, Delakas D, Zoumpourlis V, Spandidos DA. Expression profile of Rho kinases in urinary bladder cancer. J. Buon. 2011;16:511-521.

47. Griner EM, Dancik GM, Costello JC, et al. RhoC Is an unexpected target of RhoGDI2 in prevention of lung colonization of bladder cancer. Mol Cancer Res. 2015;13:483-492.

48. Kamai T, Tsujii T, Arai K, et al. Significant association of Rho/ ROCK pathway with invasion and metastasis of bladder cancer. Clin Cancer Res. 2003;9:2632-2641.

49. Zaravinos A, Volanis D, Lambrou GI, Delakas D, Spandidos DA. Role of the angiogenic components, VEGFA, FGF2, OPN and RHOC, in urothelial cell carcinoma of the urinary bladder. Oncol Rep. 2012;28:1159-1166.

50. Xie S, Zhu M, Lv G, et al. Overexpression of Ras homologous C (RhoC) induces malignant transformation of hepatocytes in vitro and in nude mouse xenografts. PLOS ONE. 2013;8:e54493.

51. Zhao Y, Zong ZH, Xu HM. RhoC expression level is correlated with the clinicopathological characteristics of ovarian cancer and the expression levels of ROCK-I, VEGF, and MMP9. Gynecol Oncol. 2010;116:563-571.

52. Golen KL, Bao LW, Pan Q, Miller FR, Wu ZF, Merajver SD. Mitogen activated protein kinase pathway is involved in RhoC GTPase induced motility, invasion and angiogenesis in inflammatory breast cancer. Clin Exp Metastasis. 2002;19:301-311.

53. Shao R, Zhang FP, Tian F, et al. Increase of SUMO-1 expression in response to hypoxia: direct interaction with HIF-1alpha in adult mouse brain and heart in vivo. FEBS Lett. 2004;569:293-300.

54. Bae SH, Jeong JW, Park JA, et al. Sumoylation increases HIF1alpha stability and its transcriptional activity. Biochem Biophys Res Commun. 2004;324:394-400.

55. Li Z, Wang D, Messing EM, Wu G. VHL protein-interacting deubiquitinating enzyme 2 deubiquitinates and stabilizes HIF-1alpha. EMBO Rep. 2005;6:373-378.

56. Wu S, Kasim V, Kano MR, et al. Transcription factor YY1 contributes to tumor growth by stabilizing hypoxia factor HIF-1alpha in a p53-independent manner. Cancer Res. 2013;73:1787-1799.

\section{SUPPORTING INFORMATION}

Additional Supporting Information may be found online in the Supporting Information section.

How to cite this article: Guo Y, Wang J, Zhou K, et al. Cytotoxic necrotizing factor 1 promotes bladder cancer angiogenesis through activating RhoC. The FASEB Journal. 2020;34:7927-7940. https://doi. org/10.1096/fj.201903266RR 